\title{
Social Media and Its Impact on Chinese's Women Body Image: The Effects of Body Comparison and Motivation for Social Media use
}

\author{
Jingyi Liu \\ Annie Wright Schools, 827 North Tacoma Avenue, Washington, Unites Sates \\ *Corresponding author.jingyi_liu@aw.org
}

\begin{abstract}
Social media ingrains the lives of countless individuals in the world. With adolescents, particularly young women being the primary users of social media platforms, the question of whether social media use has an impact on their self-esteem, body dissatisfaction, body image, and self-concept. In social media, people can easily find many "perfect" body photos, and people in real life tend to believe that is the "ideal" body and develop negative influence and views on their own body. This exploratory study will conduct through the hypothesis that women develop negative body images after viewing the perfect body images promoted by social media with further experiments.
\end{abstract}

Keywords: social media, Body Image, Body Comparison, Social Comparison Theory, Body Dissatisfaction

\section{INTRODUCTION}

Social media includes books, magazines, podcasts, movies and so on, which is an immense range. The influence on body image is always debatable, most of the researchers think its influence on body image, but some hold an opposing opinion. Researchers consider it to have a positive effect. Also, research cultural background still plays an essential factor. Therefore, it is necessary to investigate under one specific culture, with a particular group.

In the western world, there was a lot of research on body image. Researchers investigate and analyze body image in psychology, sociology, media, and clinical factors. They proposed that body image is a psychological and behavioral characteristic of individuals; it is affected by socio-cultural factors and produced by the interaction between individuals and social culture. They proposed that body image is psychological and behavioral characteristics of individuals; it is affected by socio-cultural factors and produces by the interaction between individuals and social culture. Studies show that exposure to the "ideal body" female model image in mass media significantly affects women's cognition of the ideal body shape, which is closely related to body image, eating disorder, slimming intention, and behavior[7]. The research on body image in the Taiwan region of China shows that the ideal female image in mass media will negatively impact female body image. However, in

mainland China, the influence of female body image is still in its infancy, and there are few related studies.

There are few scholarly articles about media's influence on women's bodies in social psychology. Culture shapes the context in which body image is formed, and hence its uniqueness in different sociocultural environments [10]. Due to the differences between Chinese and Western cultures, it is necessary to conduct localized research on whether western research results apply to national conditions.

Through a self-based survey, the possible correlation between social media use and negative body image was found. The results can serve as evidence for negative body image caused by the "Ideal body" caused by social media promotions. Also, people can reflect the influence of social media on women's view of their bodies from a social psychology perspective and provide a theoretical theory to cultivate a positive body image for women.

\section{ANALYSIS}

\subsection{Media influences on the negative effect of the body image}

Body image is how you feel your body is 
aesthetically and how attractive you perceive yourself (Nordqvist, 2012). In other words, it is the impression of people's concept of themselves. These signs are obsessing to observe the body in the mirror in a selfexamine way with self-dissatisfaction and frequently compare the shape and size of one's own body to other people's bodies. Other signs may also include comparing own body to a celebrity or friend's body. Negative body image is often measured by the level of selfdissatisfaction and is attributable to a discrepancy between the perception of body image and idealized body image [8].

Social media objectifies the female body, leading women to view their bodies as objects that can be changed. Social media objectifies the female body, which leads women to view their bodies as an object that can be changed. They focus more on the outside instead of the internal body function and lead towards comparing their bodies. Specifically, the media consistently and statically portrays the body. For example, one research about the popular magazine on men and women's activity analysis shows men moves far more than women, where women maintain more static. The way that magazine portrays may imply women's body as an object through decoratively depicting their body instead of expressing the function of their body. In the advertisement, the objectification of women's bodies is even more vital. For instance, Women's bodies are often depicted as smooth chocolate, thighs are compared to the lure, and body curves are used as a metaphor for cars' comfort level.

\subsection{Social Comparison Theory}

Social comparison theory was initially developed in the social psychology field, which serves for the research of human behavior. Festinger, Stanley Schachter, and Kurt Back co-authored "Social Pressures in Informal Groups" in 1950. In the book, Festinger and Thibaut proposed social reality, with this concept and more indepth research, and proposed social comparison theory in 1954. It is often known as the classical theory of social comparison [4]. Festinger suggested that people have an innate drive to evaluate themselves, often through comparison with others or analyzing themselves with others. Thus, there is a strong incentive for individuals to attempt to close the gap between reality and the ideal status when there is a gap between.

Nevertheless, when no objective comparison is provided, individuals compare themselves with similar individuals. This "similarity hypothesis" is the core thesis of social comparison theory, which explains how individuals choose the comparison target. Festinger proposes that when the target object does not exist, Individuals tend to compare themselves to the people like them.
The process of comparing is often unconscious and shared among people. This automatic process in real life cannot arouse the awareness of women comparing the ideal body on social media with themselves. In addition, even if they are aware, they may be reluctant to reveal true thoughts because of social expectations. Models in social media are much thinner than women in the real world, so the comparison with skinny models is usually upward comparison, explaining why exposure to the ideal images in the media can lead to negative body image.

\subsection{Types of Social Comparison theory}

There are two major types of social comparison theory: upward comparison and downward comparison. Upward comparison is where people compare themselves to people who are better than them. When individuals evaluate their abilities, upward comparisons bring out a self-improvement motivation, contributing to self-reflection. Studies show that social comparison about the body tends to be upward comparisons rather than downward comparisons, and upward comparisons reduce an individual's self-confidence, resulting in less attractiveness and negative body image.

A downward comparison is when people compare themselves to less proficient people to maintain selfesteem and subjective well-being. Downward comparison theory develops the classical social comparison theory in both motivation and direction of comparison. According to this theory, when individuals encounter failures and such adverse events, their selfesteem and mental health will decline. Currently, individuals tend to compare with others who are worse than themselves to maintain self-esteem and subjective well-being.

\subsection{Motivation of social comparison}

There are three primary motivations for social comparison, self-evaluation, self-improvement, and selfsatisfaction. Many researchers agree with this concept; in Trope's experiment and the participants were given a set of missions, they could choose one. As a result, the participants select the mission that can test their abilities, even if the results are not desirable. Festinger mainly focuses on two points of motivation for self-evaluation: abilities and perspectives.

According to Festinger, people tend to compare with similar people--the less similar it gets the less willing they are to compare. By similarity, he means the evaluated dimension, such as abilities and opinions. However, when individuals conduct self-evaluation, they will not limit the social comparison to the evaluated dimension but also consider those related dimensions to conduct a better and richer comparison. Zanna mentioned an example. Swimmers are asked to rate their 
swimming speed, and when selecting a target for comparison, they consider the speed of other swimmers (the evaluated dimension) and their age, experience, and recent training (the dimension related to swimming speed). He/she will not necessarily be compared to someone who swims at speed like their own, but to someone who swims at speed like his/her own on a relevant dimension. Therefore, it is necessary to distinguish between similarity in evaluation dimension and appropriate dimension. Also, peers play a crucial part in making sense of images and messages from social media and provide comparison targets themselves.

To sum up, in the process of social comparison driven by self-evaluation, people are more willing to compare with others who accurately grasp their relative status. The similarity includes the similarity in the evaluated dimension, the similarity in the related attribute, and the unrelated dimension. Depending on the contest of the comparison, different comparison appraisals lead to different results, it could be deflecting threats or learning from others and evaluating. This could result in a negative body image developed by comparing through the better body and emerge lack of self-perception and confidence. Also, a better understanding of the use of comparisons to counter threats could end better, promoting self-motivation through body comparison that led people to try harder to accomplish a better body.

\subsection{Self-improvement and social comparison}

Upward comparisons promote self-improvement. People tend to observe and learn from more capable or successful people and inspire themselves by their example. Research on imitation has shown that people are most likely to imitate the behavior of others like themselves and to adopt similar performance standards. Being like the results of others is meaningful to us because it allows us to infer our results or potential. Similarity includes not only the similarity of related attributes but also the similarity of unrelated attributes.

Upward comparisons are also risky, where it forces people to face their self-abasements. Wheeler argues that highly motivated people are most likely to enjoy upward comparisons, and they rarely experience low self-esteem because they see themselves as those who excel and deduce that they will succeed one day. Therefore, they will explore a motivation to achieve better, which is positive for body image. Nevertheless, upward comparisons can be especially painful if people are close or like themselves on relevant dimensions.

Downward comparisons can reduce stress or boost self-esteem, which results in positive body image. However, exposure to danger may not be a prerequisite for downward comparisons, which are self-reinforcing even when people are not threatened.

\subsection{Social comparison orientation}

Gibbons and Bunk [10] first proposed the concept of social comparison orientation. Individuals with high social comparison tendency often compare themselves with others and are easily affected negatively by social comparison. When making a downward comparison, individuals with high social comparison may have a bad self and feel that the comparison goal reflects their situation, leading to damaging effects. According to research, individuals with high social comparison tendencies are more likely to develop a stronger sense of uncertainty about themselves, which is more likely to develop body image. There is a scale conducted by Gibbons and Bunk that is used to measure social comparison tendency based on social comparison orientation. It is composed of eleven questions, divided into two factors of ability and concept structure that are used to study social comparison characteristics related to the physical appearance of the participants.

\subsection{Social culture theory and its impact of social media on body image}

The sociocultural theory was put forward by the former Soviet Union psychologist L.S.Vygotsky. It emphasizes that social and cultural factors play a central role in developing human cognitive function and studies how social and cultural values affect individual values and behavior. The sociocultural theory holds that cultural values are essential to how individuals perceive themselves and how individuals are perceived. Sociocultural theory is often used to explain how negative body images are formed under the influence of mass media.There are three primary models that social media has its influence on women's view of their bodies. When the concept "the skinnier, the better" is maintained and developed among the family and peers, teenagers tend to develop and believe this concept. Under the influence of the social environment, it is vital to be "skinny" everywhere; diet and weight control are likely to lead to eating behavior disorders and negative body image. Stice proposed a dual model that when inappropriate information such as "you are not skinny enough" or criticism related information in the mass media is tolerated and reinforced by family and peers, and when this happens, individuals develop poor selfconcept and low self-esteem, individuals are prone to developing negative body image. A final theoretical view is that the social comparison process is a mechanism that links media exposure, media stress, and increased internalization of media values to physical dissatisfaction and negative body image. Internalization of the "skinny" is a core concept of sociocultural theory, which refers to women internalizing the sociocultural standard that "skinny is beautiful," resulting in negative body image. 
Internalization is when an individual agrees with the media's message and accepts social and cultural standards about physical appearance. The degree of internalization will affect the individual's attitude and intention towards their own body. Individuals with a high degree of internalization are more likely to compare the ideal image in the media and take practical actions (such as losing weight, cosmetic surgery, dieting.) to change their appearance and body shape to meet social and cultural standards. If they do not, mental health problems such as depression, anxiety, body image problems, and eating disorders can occur.

\section{RESEARCH}

A survey based on the social comparison orientation stated by Gibbons. The participants are purposely informed that this survey is about media use, not the social comparison, to maintain objectiveness. This survey will determine the tendency of comparison to others and its relationship with negative body image. Participants are asked to rate ten different questions on a scale of 1-5; the higher the rating, the higher they agree with the questions.

\subsection{Analysis}

Table 1 Time spent on social media vs scoring in the survey

\begin{tabular}{|c|c|c|c|}
\hline $\begin{array}{c}\text { Time spent } \\
\text { on } \\
\text { Social media } \\
\text { (hrs) }\end{array}$ & Samples & Average & $\begin{array}{r}\text { Standard } \\
\text { Deviation }\end{array}$ \\
\hline $1-5$ & 17 & 2.35 & 1.17 \\
\hline $5-10$ & 27 & 2.67 & 1.04 \\
\hline $10-15$ & 12 & 3.00 & 1.21 \\
\hline $15-20$ & 9 & 3.00 & 1.00 \\
\hline Others : & 4 & 4.00 & 1.41 \\
\hline F & & 2.085 & \\
\hline p & & 0.093 \\
\hline
\end{tabular}

As shown in figure 1, the higher the time the participants spend on social media, the higher the score in the test. For people who use social media for one to five hours, their average score is 2.35 , where they are less likely to compare themselves with the people on social media. For people who spend fifteen to twenty hours on social media, their average score is reaching up to 4 , where they are most likely to compare themselves.

This survey investigated the effects of social comparison orientation on individuals' tendency to compare in social media. It also examined the multiple parallels and sequential relationship between social comparison orientation and women's body image. The results showed that social comparison orientation negatively affected body image. Given the tendency toward upward social comparison in social media, the study participants were likely to experience negative feelings toward other social users who seemed to have a better body.

The comparison between women and "skinny ideal" models is upward comparison. When women with high social comparison tendency are in the upward comparison, their self-evaluation motivation may be dominant; when women gain the gap between their own body and the model's body, their self-evaluation decreases, which leads to more negative body image.

The survey results on the mass media and female body image show that exposure to the "skinny" female model image in social media harms the body image of females, the core of which lies in the internalization of the "skinny" ideal body image. If they are not exposed to social normative information, neither overweight nor thin women will be dissatisfied with their body image. However, after receiving the social normative information about "skinny is beautiful," some women will negatively affect their body image. The way that women will receive social normative information is from social media, which highlights the result that the more time women spend on social media, the more possibilities they will develop body image.

\section{CONCLUSION}

In general, the survey and research demonstrated that social media plays a crucial role in influencing Chinese women's negative body image. By applying social comparison theory, most people are not aware they are comparing themselves to others. Thus, social media promotes the ideal women's body in an objectification way, where women are viewed as objects that serve as visual entertainment.

There was a significant positive correlation between media attention and body image, confirming the study's hypothesis, which proves the hypothesis that women develop negative body image after viewing the perfect body images promoted by social media. Media attention has a significant impact on body image. When individuals pay attention to the media about fashion, appearance, and other information, they feel pressured to change their appearance, feel dissatisfied with their appearance, and thus generate negative body image.

\section{REFERENCES}

[1]Cusumano,D.L., \& Thompson,K.J.(1997). Body image and body shape in magazines,exposure, awareness, and internalization. Sex Roles, 37(9/10)701-721. 
[2]Devendra,Singh.(1995).Female Judgment of Male Attractiveness Relationships:Role of waist-to-hip Ratio and financial and Desirability for status.

[3]Fallon, A.(1990). Culture in the mirror: Sociocultural determinants of bodyimage. New York: Guilford Press.

[4]Festinger,,L.(1954).A theory of social comparison processes. HumanRelations, 7,117-140.

[5]Furnham,A., \& Greaves,N.(1994). Gender and locus of control correlates of body image dissatisfaction. European Journal of Personality, 8,183-200.

[6]Goethals, George R. "Social Comparison Theory." Personality and Social Psychology Bulletin, vol. 12, no. $3, \quad 1986, \quad$ pp. 261-278., doi:10.1177/0146167286123001.

[7]Grogan, Sarah, and Sarah Grogan. "Body Image." 2007, doi:10.4324/9780203004340.

[8]Hosseini, Seyed Alireza. Body Image Distortion, 2021.

[9]S, Kesici, and Erdogan A. Mathematics Anxiety According to Middle School Students' Achievement Motivation and Social Comparison, 2010.

[10] Buunk, Abraham P., and Frederick X. Gibbons. "Social Comparison Orientation: A New Perspective on Those Who Do and Those Who Don't Compare with Others." Social Comparison and Social Psychology, pp. 15-32., doi:10.1017/cbo9780511584329.003.

[11]Yam, Mei. Does Culture Matter in Body Image? The Effects of Subjective and Contextual Culture in Body Image among Bicultural Women., 2013.

[12]Personality and Social Psychology, 69(6),10891101. 